\title{
Illumination Balancing Algorithm for Smart Lights
}

\author{
Muhammed Taha Koroglu and Kevin M. Passino, Fellow, IEEE
}

\begin{abstract}
In this paper, we present two decentralized algorithms that aim to achieve uniform lighting across the floor of an experimental testbed under a variety of challenges, including cross-illumination effects and external light disturbances. These challenges cause over-illuminations in the environment that result in a waste of energy and discomfort to the occupants. First, a decentralized integral control approach that does not have any communication between the lights is developed and applied to the system. Due to its failure in achieving uniform lighting when the cross-illumination effects are maximized, a new decentralized method called the illumination balancing algorithm (IBA) is developed that takes the local light levels into account when adjusting the light voltages. The stability analysis of the IBA for the full height partitions case of the testbed is shown as well as the regulation problem results where the algorithm successfully balances the illuminations and hence achieves uniform lighting. In order to track a desired light level across the zones, the IBA is augmented with an integral control at an arbitrarily selected control loop. This combined algorithm achieved successful control even in a case where the decentralized integral control failed.
\end{abstract}

Index Terms-Decentralized, load balancing, smart light control system, illumination balancing algorithm (IBA).

\section{INTRODUCTION}

$\mathbf{E}$ NERGY conservation has become a crucial research topic due to the increasing demand for energy in today's developing world. Exhaustion of energy resources and considerable environmental issues (e.g., ozone depletion, global warming) point to the necessity of research that aims to avoid energy waste. Among various components of overall energy consumption, lighting represents a major one. It consumes close to $15 \%$ and $35 \%$ of the electricity used in residential and commercial buildings, respectively, in the U.S. [1], [2]. Along with the energy usage of $11 \%$ in industry [3], lighting uses about $18 \%$ of the electricity. Commercial buildings account for close to $71 \%, 18 \%$ of which is overall lighting electricity use. For this reason, the smart lighting applications mostly focus on commercial buildings.

Automatic lighting controls can reduce lighting energy consumption by $50 \%$ in existing buildings [4], [5]. These lighting control systems gained popularity in recent years as they pay for themselves quickly due to considerable reduction in energy usage. The energy saving is provided by avoiding redundant illumination (i.e., over-illumination). Over-illuminations occur due to multiple artificial lights in the ceiling and/or daylight penetrating the room. In a shared-space office, the light illuminates not only the cubicle under it but also rest of the

Manuscript received May 14, 2012; revised October 18, 2012 and February 8, 2013; accepted April 4, 2013. Manuscript received in final form April 13, 2013. Date of publication May 17, 2013; date of current version February 14, 2014. Recommended by Associate Editor Z. Gao.

The authors are with the Department of Electrical and Computer Engineering, The Ohio State University, Columbus, OH 43210 USA (e-mail: m.tahakoroglu@gmail.com; passino@ece.osu.edu).

Digital Object Identifier 10.1109/TCST.2013.2258399 cubicles at different levels depending on the distance from the light to the cubicle. The contribution of a light to the light level of the other cubicles is the cross-illumination effect of the particular light. When these cross-coupling effects arise, lighting control requires communication between the lights in order to obtain a desired light level across the room.

Wireless sensor networks (WSN) are used in some lighting applications. They provide global communications between the lights. The nodes send the data to a central controller (i.e., centralized approach) in a WSN. A smart lighting problem in a shared-space office is formulated into a linear programming problem under the assumption that the light level at a particular point in the environment is the summation of light from each luminaire in [6] and [7]. The problem is solved with a centralized control strategy by using a WSN.

In addition to cross-illuminations, external daylight is another challenge in lighting control. The lights take advantage of natural light in order to use the least amount of energy necessary to uniformly light a room. The concept of utilizing sunlight for illumination is called daylight harvesting. When integrated with photosensors, the system saves considerable energy by attenuating artificial lights in response to daylight. The centralized control strategy developed in [8] adapts to the presence of natural light and provides a significant reduction in electric light usage. The authors investigate how a WSN can be used to adjust the lights to illuminate only the spaces receiving inadequate natural light in [9].

Some lighting control systems take occupant preferences into account and hence provide maximum comfort to users. The research in [10] focuses on occupant satisfaction and acceptance in office buildings with different structures. All offices have daylight responsive systems and daylighting (the use of a control system to modulate artificial light levels in response to daylight) is studied in terms of user reactions and energy savings. User preferences are considered constraints in the linear programming approach in [6] and [7].

The smart lighting systems that have the ability to tune the light levels focus on avoiding over-illumination to save energy. These systems can also detect under-illuminations that might occur when the daylight intensity decreases during the day (e.g., disappearance of the sun when the weather is cloudy), and tune the artificial lights. Moreover, some of the systems are equipped with sensors (e.g., ultrasonic sensors, passive infrared sensors, cameras) that can detect if a part of the room is not occupied for some time [11]. The system can shut down the lights that are responsible for the illumination of the unoccupied areas. The combination of these two functionalities (i.e., tuning the light levels and turning the lights ON/OFF) results in the highest energy savings in buildings.

In addition to energy savings, some evidence indicates that exposure to daylight reduces stress [12]. A study of the effect 


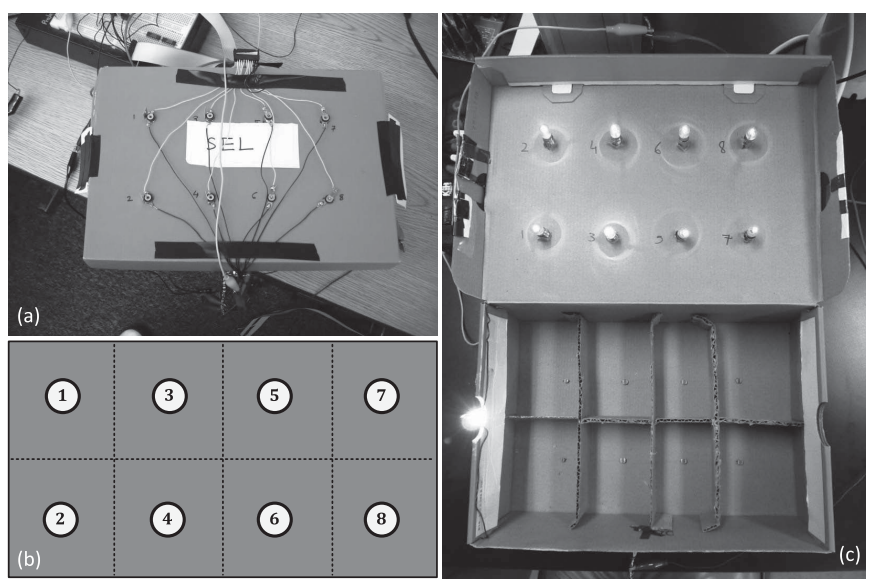

Fig. 1. (a) Box viewed with its lid closed. (b) Zone layout from top view. (c) Interior of the box. A photocell is placed at the bottom of each zone. Half height cardboard partitions are shown in this picture, which partially isolate the zones from each other.

of daylighting on sales performance is given in [13]. Results have shown that there is an important relationship between daylight availability in buildings and human factors that affect sales performance. Benefits of daylighting are studied in [14].

In comparison to all the above-mentioned research on lighting controls ours is novel in that it provides a distributed algorithm [the illumination balancing algorithm (IBA)]. Such an algorithm can be scaled up to large-scale applications with many lights and sensors. Moreover, as we show here, it can overcome light disturbances and room structural variations (e.g., in partitions).

\section{EXPERIMENTAL TESTBED}

\section{A. Model Building and Light-Sensor Layout}

A small box is used as the testbed as shown in Fig. 1. This testbed is a physical model of residential, commercial, and industrial environments; by using cardboard partitions at different heights, the effects of multiple rooms in a building are emulated (e.g., cubicles in an office building). Eight miniature incandescent bulbs (\#1847) are placed in the top of the box as shown in Fig. 1(a) and (c). To sense the illumination levels in each zone, eight Cadmium-Sulfide photocells (\#276-1657) are fixed directly under the lights on the bottom of the box. In this way, eight zones are defined; each zone consists of a bulb and the sensor under it. An external bulb is placed at the left side of the box as can be seen in Fig. 1(c) in order to conduct daylighting experiments.

\section{B. Photosensors and Interface Circuity}

Light-dependent resistors (LDR) are utilized to sense the light levels at the bottom of the box. As its name implies, the resistance of an LDR varies when the light intensity falling on it changes. If there is no light on an LDR, its resistance is infinite; it behaves as an open circuit. When the light intensity falling on it increases, the resistance of the LDR decreases. By using this principle, the light level can be converted to a voltage signal with a voltage divider circuit. Hence, the light level will be measured in volts in the experiments although
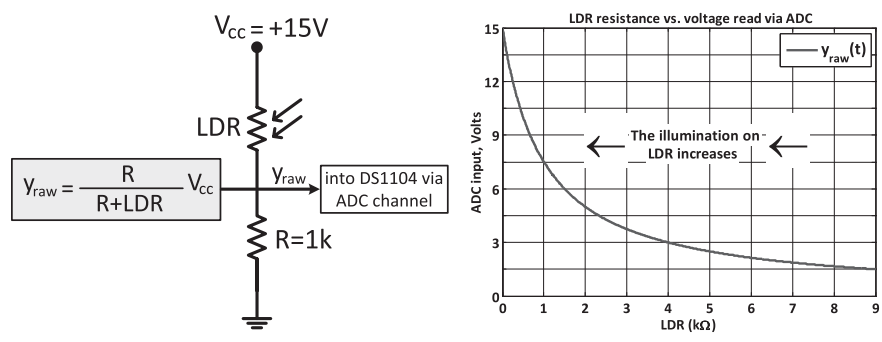

Fig. 2. Voltage divider circuit that is used as interface circuitry to the DS1104 hardware (i.e., microcontroller) and the raw sensor output according to given circuit.

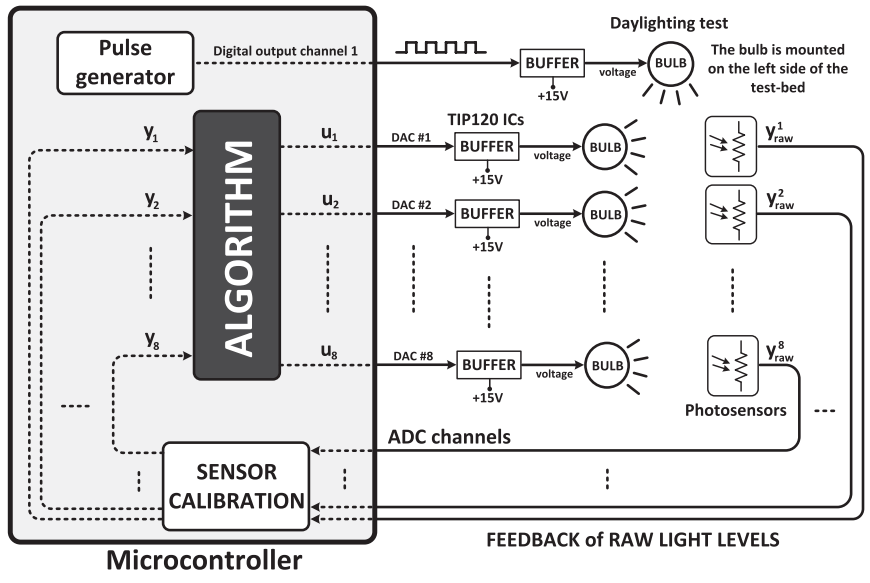

Fig. 3. Pictorial representation of the overall smart lighting system. Here, $u_{i}$ denotes the applied voltage signal to the $i$ th bulb, $y_{\text {raw }}^{i}$ and $y_{i}$ denote the raw sensor output and light level in the $i$ th zone, respectively, for $i \in\{1,2, \ldots, 8\}$.

the unit for light intensity is lux. The divider circuit along with its simulation can be seen in Fig. 2. In the figure, the raw sensor output, $y_{\text {raw }}(t)$, grows when the illumination on the LDR increases.

\section{Overall System}

The light levels should be converted to digital signals in order to be processed in the microcontroller; therefore, ADC channels of the microcontroller are used in this operation. After sensor calibration (see below) and generating the voltage signals to be applied to bulbs, the output data are converted to continuous time signals via DAC channels. In addition, one of the digital output channels is used in generating repeatable identical external light disturbance to the testbed. An overall representation of the closed-loop system is depicted in Fig. 3.

There is a tradeoff between the quality of a sensor and its cost. Due to the cheap price of the LDRs, this tradeoff is experienced in the testbed. Differences in sensor readings occurred when a voltage sweep is implemented at each zone. In addition to these differences, the threshold voltages of the transistors inside the buffer ICs caused dead zones at the beginning of the valid range (i.e., $0-10 \mathrm{~V}$ ). Eventually, the sensor calibration is made in a way such that it results in $y_{i} \approx u_{i}, \forall i \in\{1,2, \ldots, 8\}$ in the linear region of full height partitions case. In the experiments, we mostly stayed in the linear region. Nonetheless, even though the signals go to nonlinear regions, still the experiments are valid due to usage of feedback in the system. 


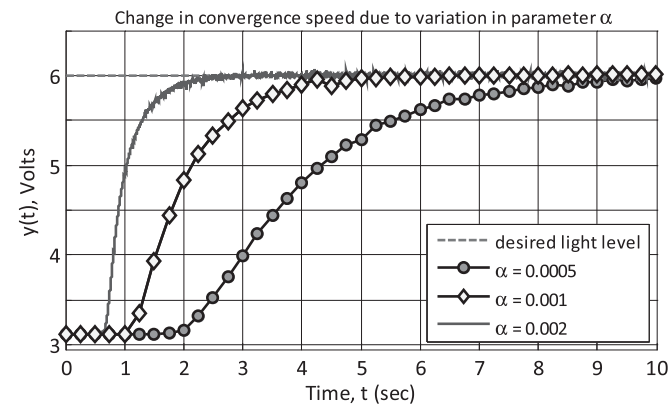

Fig. 4. Convergence to desired light level with heuristic design.

\section{Decentralized Integral CONTROL}

\section{A. Single Room Case}

1) Intuitive Design: A single room is enclosed by full height cardboard partitions and consists of one bulb and one photosensor. Due to isolation of the room from outside, there will be neither cross-illumination nor external light effects; only the bulb affects the actual light level in the room. The bulb is initialized with an arbitrary voltage signal. Subsequently, the error signal which is the difference between the desired light level and actual light level is computed. The sign of the error determines if the voltage on the bulb should be increased or decreased. If over-illumination occurs in the room, then the sign of the error will be negative meaning that the light should be dimmed. On the contrary, when the actual light level is less than the desired light level, the sign will be positive and the bulb voltage should be increased to reach the desired light level in the environment. The voltage value that should be applied to the bulb in order to obtain the desired light level will be found easily with this simple intuitive approach. The algorithm is expected to be successful even when there are external light disturbances that will be introduced to the testbed to test for daylighting performance.

2) Implementation of the Algorithm: The expression for the algorithm is given as

$$
u(k T)=u(k T-T)+\alpha e(k T-T)
$$

where $T$ denotes the sampling period, $u$ refers to voltage signal on the bulb, $e$ is for the error signal, $k T$ and $k T-T$ denote the current and the previous steps, respectively, for $k=1,2,3, \ldots$. The design parameter is $\alpha$ and it determines the speed of convergence. The algorithm will be applied to zones with a decentralized approach (i.e., there will be eight control loops). The actual light level is denoted by $y_{i}(k T)$ for the $i$ th zone while $d(k T)$ represents the desired light level for all the zones.

The algorithm converges when $\alpha>0$. When $\alpha=0$, the illumination stays constant since there are no updates on $u(k T)$. If $\alpha<0$, then the algorithm diverges. For positive values of $\alpha$, convergence speed is illustrated in Fig. 4. Note that when $\alpha$ takes greater values, the convergence speed increases dramatically. However, after some point, it causes oscillations.

Before applying the algorithm to the testbed, we look at the linear difference equation given in (1) from another point of view. It is trivial to go to $z$-domain by using the definition of the unilateral $Z$-transform given as

$$
U(z)=Z\{u(k)\}=\sum_{k=0}^{\infty} u(k) z^{-k}
$$

where $z$ is a complex number. When the $Z$-transform is applied to both sides of (1), the equation becomes

$$
U(z)=z^{-1} U(z)+\alpha z^{-1} E(z) .
$$

If this equation is manipulated, we get

$$
C(z):=\frac{\alpha}{T} \frac{T}{z-1}=\frac{\alpha}{T} F(z)
$$

where

$$
F(z):=\frac{T}{z-1}
$$

$F(z)$ is the expression for the forward Euler numerical integration method. Hence, $C(z)$ can be considered as an integrator with a gain of $\alpha / T$. Consequently, the relationship between the integral controller in control theory and the intuitive design here is

$$
K_{i}=\frac{\alpha}{T}
$$

where $K_{i}$ denotes the gain of the integral controller. Thus, it is shown that the heuristic algorithm coincides with nothing but an integral controller. The design parameter $\alpha$ can be considered as the gain of the integral controller due to proportional relationship with $K_{i}$. In the following experiments, $\alpha$ and the sampling period $T$ are selected as 0.001 . The design parameter is determined heuristically while the ability of the microcontroller along with the dynamics of the system defined the sampling period. This case (i.e., $\alpha=0.001$ and $T=0.001$ ) corresponds to an integral controller with $K_{i}=1$.

\section{B. Decentralized Integral Control: Experimental Results}

The heuristic algorithm is implemented for each zone separately for all three cases of partition heights. In this decentralized approach, the $i$ th light voltage $u_{i}$ depends on only the corresponding light level $y_{i}$ where $i \in\{1,2, \ldots, 8\}$. None of the light voltages are produced by considering the light levels of the other zones (i.e., there is no communication between the lights).

1) Full Height Partitions: Full height partitions case is actually trivial as the cardboard partitions isolate all eight zones completely from each other; it is expected that all the light levels converge to desired light levels similar to Fig. 4. Yet the experimental results are shown in Figs. 5 and 6 $(\alpha=0.001)$ to complete an overall picture of the testbed. All light levels converged to desired light level quickly. We observed $y_{i} \approx u_{i}, \forall i \in\{1,2, \ldots, 8\}$ in the linear region as a consequence of the sensor calibration.

2) Half Height Partitions: In this case, due to the reduction in heights of the cardboard partitions, cross-illuminations affect all the photosensors (i.e., each light affects all the sensors). Note that half height partitions case corresponds to a shared-space office in real life due to partial isolation created by the cardboard partitions. Results are shown in Figs. 7 and 8 $(\alpha=0.001)$. 


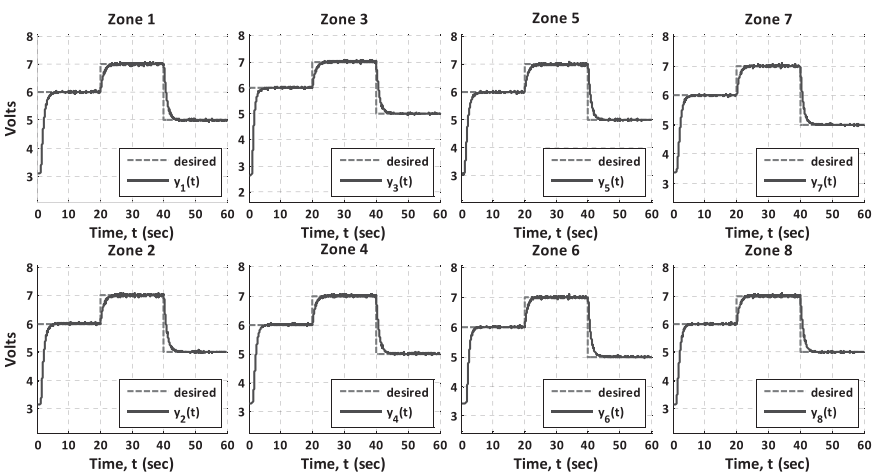

Fig. 5. Actual light levels (full height partitions case).
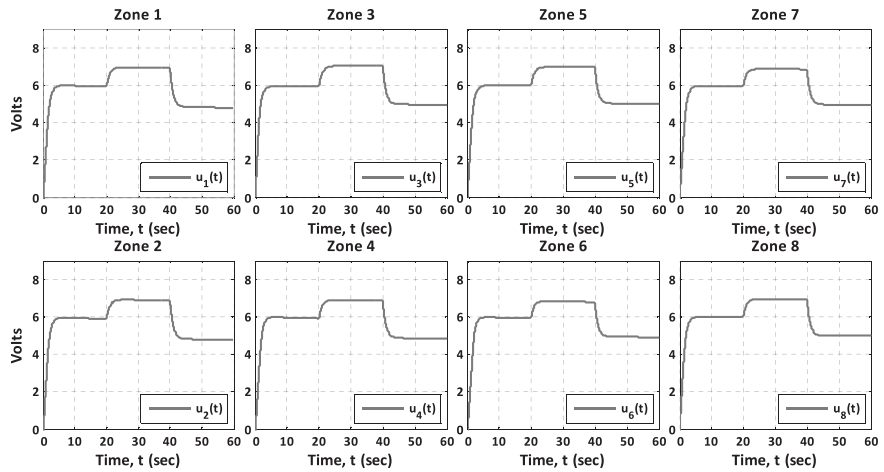

Fig. 6. Voltage signals applied to bulbs (full height partitions case).

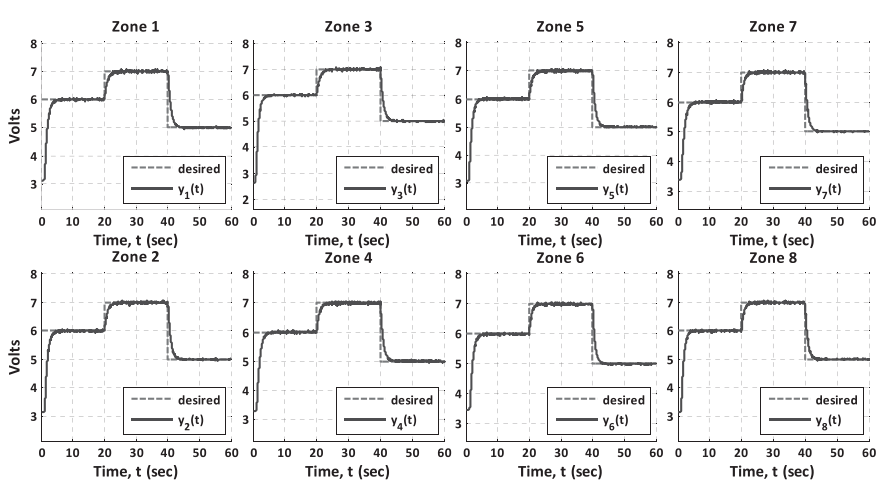

Fig. 7. Actual light levels (half height partitions case).

Similar to the full height partitions case, actual light levels quickly converged to desired light level in all the zones. The decentralized integral control became successful in reaching uniform illumination under restricted cross-illumination effects. Notice that there is a drop in steady state bulb voltage signals that result in desired uniform illumination (i.e., solutions) when Figs. 6 and 8 are compared. This reduction makes sense as now the illumination in a zone is a combination of all lights (i.e., $y_{i}=F(u)$ where $u=\left[u_{1}, u_{2}, \ldots, u_{8}\right]^{T}$, $i \in\{1,2, \ldots, 8\})$.

3) Half Height Partitions (Daylighting): In this section, in addition to cross-illumination effects, an external light disturbance is introduced to the testbed from left side. Naturally, this light will affect the light levels of the zones located at the left of the testbed more than the others. As a disturbance, pulse signals are generated in the microcontroller that turns

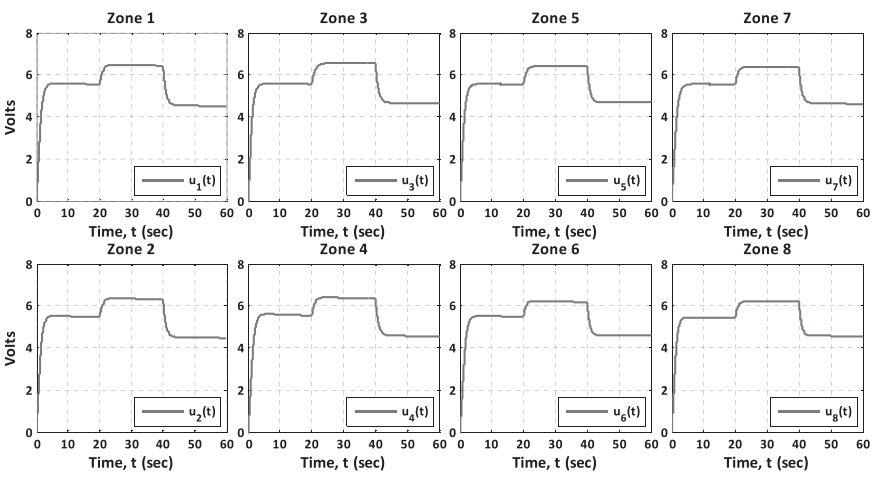

Fig. 8. Voltage signals applied to bulbs (half height partitions case).

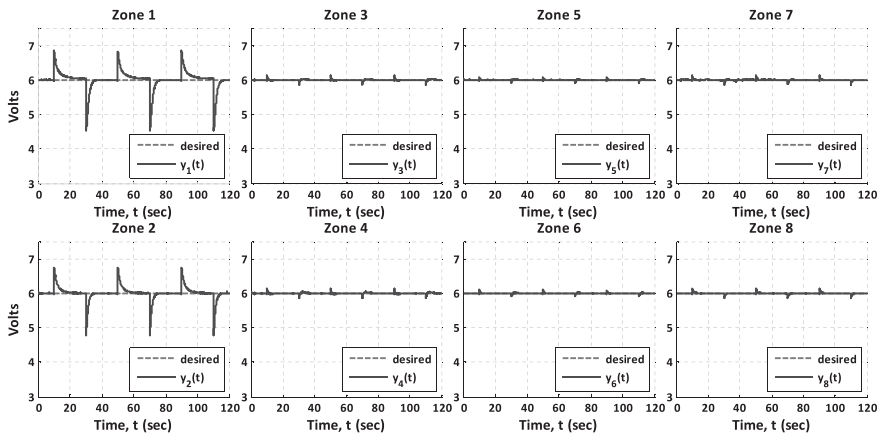

Fig. 9. Actual light levels (daylighting).

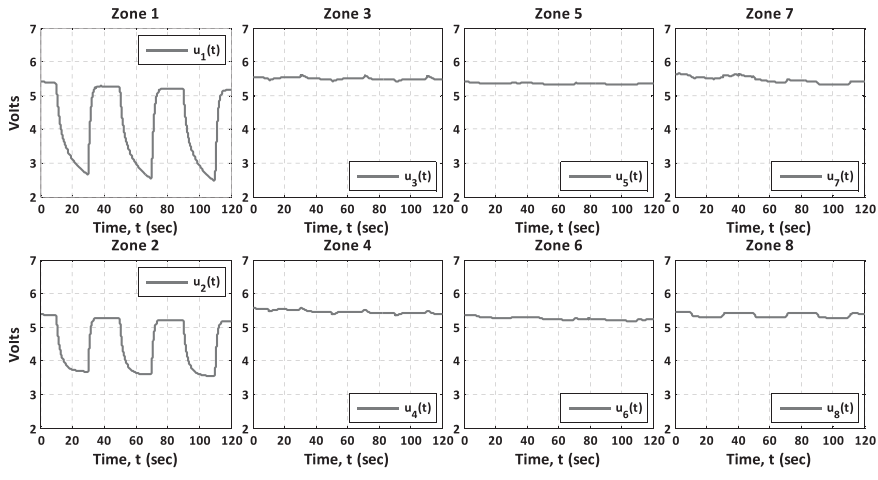

Fig. 10. Voltage signals applied to bulbs (daylighting).

the external light $\mathrm{ON}$ at $t=10,50$, and $90 \mathrm{~s}$, and $\mathrm{OFF}$ at $t=30,70$, and $110 \mathrm{~s}$, respectively.

Desired light level is 6 for all the zones throughout the experiment. The results are given in Figs. 9 and 10. Whenever the external light is turned on, over-illuminations occurred for a while. The algorithm reacted to over-illumination by reducing the voltage signals on the bulbs, and hence the light level converged back to desired illumination level. Similarly, when the external light is removed, suddenly the actual light level became less than the desired level (i.e., under-illumination), and bulb voltages are increased by the algorithm. Decentralized integral control became very successful in rejecting the light disturbances.

The energy usage is reduced when external light is present inside the testbed. In reality, more energy can be saved depending on the window size at the walls. In modern buildings, architects now take the daylight into account when designing 


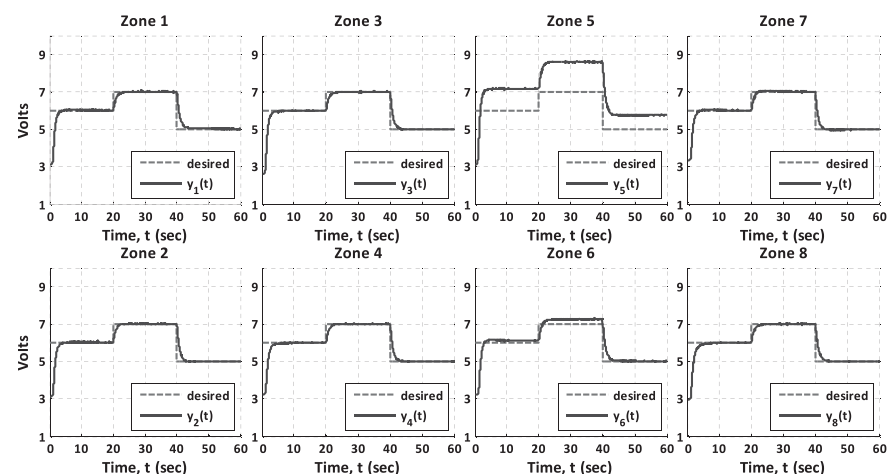

Fig. 11. Actual light levels (no partitions case).

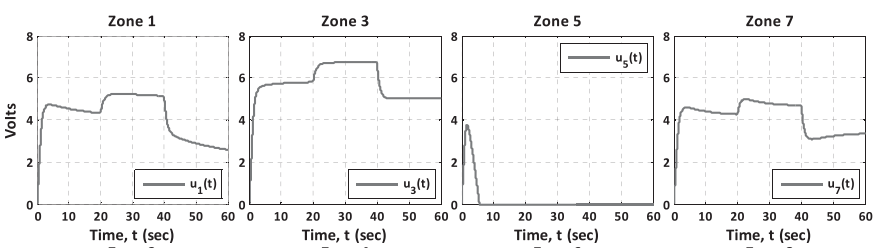
Zone 2

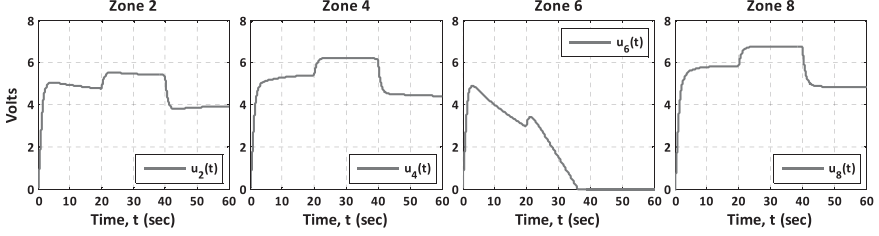

Fig. 12. Voltage signals applied to bulbs (no partitions case).

room structures. Utilizing natural light as much as possible in illuminating the environments will result in huge energy savings.

4) No Partitions: Now, half height cardboard partitions are removed and hence the effects of cross-illuminations are maximized. This will yield intensive interactions between individual control loops. Results are shown in Figs. 11 and 12 $(\alpha=0.001)$.

All zone light levels except Zones 5 and 6 converged to desired light levels. Zones 5 (obviously) and 6 (slightly) are over-illuminated, despite the fact that corresponding bulb voltages dropped to zero. This unsuccessful performance proves how crucial the cross-illumination effects are. The light level in Zone 5 converged to the desired light level much quicker than the others. The corresponding control loop started to dim the light in Zone 5 right after a little over-illumination occurred in there. However, dimming the light could not pull down the actual light level to the desired level due to excessive crossillumination effects on Sensor 5 . At $t \approx 3$, the light level in Zone 6 reached the target illumination and a similar response occurred in Zone 6 too. It took more time for bulb 6 to drop to zero as the light level in Zone 6 always stayed very close to other light levels. However, the control loop of Zone 6 could not prevent over-illumination as well. Bulbs are turned off after some time and actual light levels remained higher than the desired light level.

The results of a voltage sweep experiment in Zone 1 are illustrated in Fig. 13 to understand the reason for the failure. The cross-illumination effects increased significantly when half height partitions are removed. Especially in the

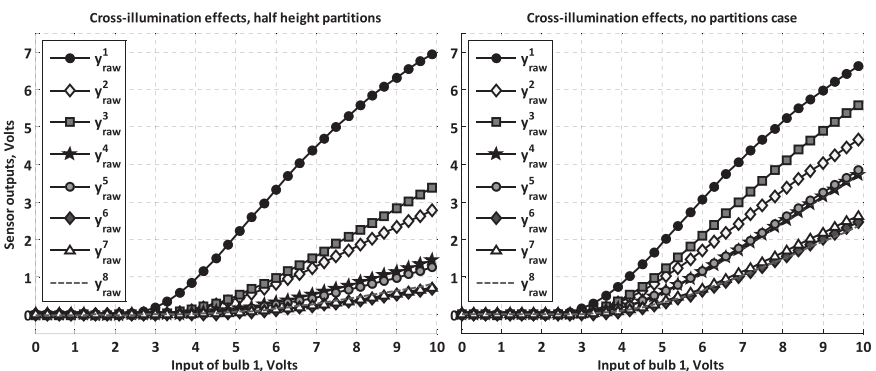

Fig. 13. Increment in the cross-illumination effects.

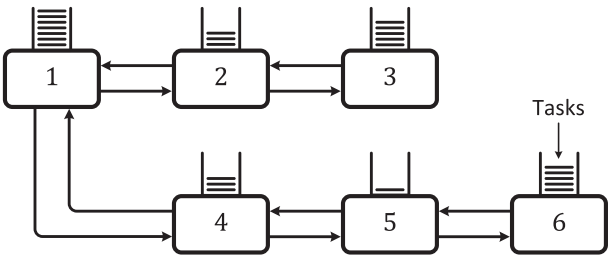

Fig. 14. Network of processors for executing tasks [16].

adjacent zones (i.e., Zones 2 and 3), the sensor readings are very close to Sensor 1 , which indicates excessive interactions between the zones. Unpredictable intensive interactions will always occur between the individual control loops with any decentralized approach that does not have communications between the lights. In order to overcome the negative effects of these interactions, using other zone light levels as well as the corresponding light level in adjusting a light voltage might be useful. It is desired to minimize the light level information used in separate control loops. Notice that if the local communications cannot overcome the cross-illumination effects, then a global communication between the lights (i.e., a centralized approach) may be required in achieving uniform lighting.

\section{Illumination BALANCING Algorithm}

In the previous section, the decentralized integral control failed in the no partitions case. The analysis of the light levels revealed that the cross-illumination effects became a major issue that has to be considered in algorithm design. This section introduces a new approach that takes these coupling effects into account by using light level information of the surrounding zones in tuning the bulb voltage signals. Local communications between the bulbs will be used to counteract the cross-illumination effects and hence to achieve desired uniform lighting across the testbed.

\section{A. IBA Formulation}

The IBA is inspired by the load balancing in processor networks problem [15], [16]. In the load balancing problem, the system consists of multiple processors running in parallel. Each processor has its own buffer in which computational tasks are queued and the processors cooperate in the execution of the tasks. The aim is to avoid under-utilization of processors by passing load between them. A processor network is depicted in Fig. 14. 


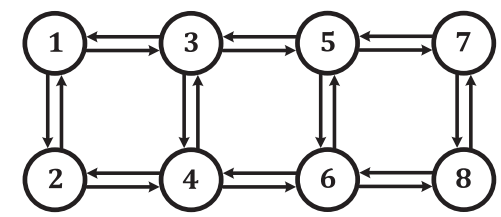

Fig. 15. Topology used in the IBA. Notice that half of the zones (i.e., Zones $1,2,7,8$ ) have two neighbors and the others have three.

In Fig. 14, an arrow starting from the $i$ th processor and terminating at the $j$ th processor means that the $i$ th processor can sense the load level of the $j$ th processor, and pass load to it if its neighbor is less heavily loaded. As there is always another arrow starting from the $j$ th processor and terminating at the $i$ th processor, both sides can pass load to each other when there is an imbalance between the load levels. Naturally, there will be no load pass when the neighbor processors are equally loaded. Notice that processors might be passing and receiving load at the same time since there can be multiple neighbors (e.g., Processors 1, 2, 4, 5 have two neighbors). The change in the load level of a processor is the sum of these transfers.

The design problem in the processor network is how much load should be transferred between the neighbors at each step. Since load levels of neighbors are compared at every step, it is possible to make the amount of load transfer between neighbors is proportional to the difference between the individual load levels. In this way, the load transfer will be big when the difference between the load levels is big (in order to balance the load as fast as possible). Also, there will be small transfers when the load levels become close in size.

When the problem is switched to the smart lights problem, the first issue that should be considered is the choice of topology (i.e., how the lights should be interconnected). As can be seen in Fig. 1, the zone layout is appropriate for a variety of neighborhood definitions in the testbed. However, actually, it is the cross-illumination effects (see Fig. 13) that should be taken into account in determining the topology due to the fact that these coupling effects provide the essential neighborhood information. When this fact is considered, the network given in Fig. 15 is used for the testbed. It is seen in this figure that the local communications are compatible with the physical neighborhoods.

In the load balancing algorithm, the transferred load variable is also the affected variable. Explicitly, some amount of tasks are transferred between the processors, and it is the number of tasks that is desired to be balanced. On the other hand, in the smart lighting problem, there are two different variables; light voltage signals and the actual light levels. It is the light levels that we want to balance (uniform lighting), however there is no direct way of passing illumination from one zone to another. At this point, a fact is utilized in applying the idea of load balancing to the smart-lights testbed. When the voltage signal on the $i$ th bulb varies, the most affected sensor is the one directly under it (i.e., $i$ th sensor) for all three partition cases in the testbed where $i \in\{1,2, \ldots, 8\}$. This information is acquired by voltage sweep experiments in the zones, and an example for this is Zone 1 voltage sweep given in Fig. 13. Hence, it becomes reasonable to increase (decrease) the $i$ th bulb voltage when $i$ th zone is under-illuminated (overilluminated). The light levels are compared, but the voltage signals on the bulbs are transferred between the zones. This is the prime distinction from the original problem; here, the transferred variable (i.e., voltage signals on the bulbs) is not the affected variable (i.e., light levels). The IBA has one more difference from the load balancing algorithm. In the load balancing problem, the sum of load transfers, and the load level change in the $i$ th processor are the same. On the other hand, in the smart lighting problem, the total change in the $i$ th bulb voltage, and the corresponding light level variation due to this change are approximately equal in the full height partitions case while these two variations are definitely not equal to each other in the half height and no partitions cases.

\section{B. IBA: Stability Analysis}

1) Full Height Partitions Case Model: The zones, $Z=\{1,2, \ldots, N\}$, are all connected to a network (see Fig. 15) along which the bulbs in these zones can pass voltage to their neighbors. The network of zones is described by a directed graph, $(Z, A)$, where $A \subset Z \times Z$. For every $i \in Z$, there must exist $(i, j) \in A$ in order to assure that every zone is connected to the network, and if $(i, j) \in A$ then $(j, i) \in A$. Bulb $i$ can only pass a portion of its voltage to bulb $j$ if $(i, j) \in A$. Finally, if $(i, j) \in A$, then $i \neq j$.

We begin by specifying the discrete event system (DES) model. Let $\mathcal{Y}=\mathbb{R}^{N}$ be the set of states and $y_{k}=\left[y_{1}, y_{2}, \ldots, y_{N}\right]^{T}$ and $y_{k+1}=\left[y_{1}^{\prime}, y_{2}^{\prime}, \ldots, y_{N}^{\prime}\right]^{T}$ denote the states at times $k$ and $k+1$, respectively. Let $y_{i}\left(k^{\prime}\right)$ denote the light level at zone $i \in Z$ at time $k^{\prime}$. Let $e_{\alpha(i)}^{i, p(i)}$ represent that zone (i.e., bulb) $i \in Z$ passes voltage to its neighbors $m \in p(i)$, where $p(i)=\{j:(i, j) \in Z\}$. Let the list $\left.\alpha(i)=\left(\alpha_{j}(i), \alpha_{j^{\prime}}(i)\right), \ldots, \alpha_{j^{\prime \prime}}(i)\right)$ such that $j<j^{\prime}<\cdots<$ $j^{\prime \prime}$ and $j, j^{\prime}, \ldots, j^{\prime \prime} \in p(i)$ and $\alpha_{j} \geq 0$ for all $j \in p(i)$; the size of the list $\alpha(i)$ is $|p(i)|$. For convenience, we will denote this list by $\alpha(i)=\left(\alpha_{j}(i): j \in p(i)\right)$. The amount of voltage transferred from bulb $i \in Z$ to $m \in p(i)$ is denoted by $\alpha_{m}(i)$. Let $\left\{e_{\alpha(i)}^{i, p(i)}\right\}$ denote the set of all possible such voltage transfers. Let the set of events be described by

$$
\mathcal{E}=\mathcal{P}\left(\left\{e_{\alpha(i)}^{i, p(i)}\right\}\right)-\{\emptyset\}
$$

$(\mathcal{P}(Q)$ denotes the power set of the set $Q)$. Notice that each event $e_{k} \in \mathcal{E}$ is defined as a set, with each element of $e_{k}$ representing the passing of voltage by some bulb $i \in Z$ to its neighboring bulbs in the network. Let $\gamma_{i j} \in(0,1)$ for $(i, j) \in$ $A$ represent the proportion of the light level imbalance that is sometimes guaranteed to be reduced when bulb $i$ passes voltage to bulb $j$.

Now we specify the enable function $g$ and the state transition operator $f_{e_{k}}$ for $e_{k} \in g\left(y_{k}\right)$. For all $e_{\alpha(i)}^{i, p(i)} \in e_{k}$, where $\alpha(i)=\left(\alpha_{j}(i): j \in p(i)\right)$ and for $F_{i}=F_{i}\left(u_{i}\right)=y_{i}$ it is the case that:

1) $\alpha_{j}(i)=0$ if $y_{i} \leq y_{j}$, where $j \in p(i)$;

2) $F_{i}\left(u_{i}-\sum_{m \in p(i)} \alpha_{m}(i)\right) \geq F_{j^{* *}}\left(u_{j^{* *}}+\alpha_{j^{* *}}(i)\right)$, where $y_{j * *}=\min _{j}\left(y_{j}\right)$ for all $j \in p(i)$;

3) $\alpha_{j}(i)=\gamma_{i j}\left(y_{i}-y_{j}\right)$, where $j \in p(i)$. 
If all conditions above hold, then event $e_{k} \in g\left(y_{k}\right)$. Condition 1 prevents voltage from being passed by bulb $i$ to bulb $j$ if zone $i$ is less illuminated than zone $j$. Condition 2 implies that after voltage $\alpha(i)$ has been passed, the new light level at zone $i$ must be at least as large as $F_{j^{* *}}\left(u_{j^{* *}}+\alpha_{j^{* *}}(i)\right)$ where $j^{* *}$ is the least illuminated zone among the neighbors of zone $i$. To see that this expression holds, we need to examine the amount of voltage that is passed from the $i$ th bulb to its neighbors [i.e., $\alpha_{j}(i)$ ]. In the implementation of the IBA, the amount of voltage pass from bulb $i$ to bulb $j$ is $\alpha_{j}(i)=\gamma_{i j}\left(y_{i}-y_{j}\right)$ as given in Condition 3. It is easy to see that $\alpha_{j}(i) \leq \alpha_{j * *}(i)$ as $y_{i}-y_{j} \leq y_{i}-y_{j}{ }^{* *}$ if the parameter $\gamma_{i j}$ is chosen as the same for all $j \in p(i)$ (i.e., $\gamma_{i j}=\gamma$ ). It is seen in Figs. 5 and 6 that in the full height partitions case, the bulb voltage is approximately the same as the sensor output, so $F_{i}\left(u_{i}\right) \approx u_{i}$. Thus, (ii) becomes

$$
u_{i}-\sum_{m \in p(i)} \alpha_{m}(i) \geq u_{j^{* *}}+\alpha_{j * *}(i) .
$$

We need to verify that this expression holds as it is going to be required in showing the selected Lyapunov function is a non-increasing function in the stability analysis of the system. Expand $\sum_{m \in p(i)} \alpha_{m}(i)$ in order to analyze (8) explicitly. We have

$$
u_{i}-\alpha_{j}(i)-\alpha_{j^{\prime}}(i)-\cdots-\alpha_{j^{\prime \prime}}(i) \geq u_{j^{* *}}+\alpha_{j^{* *}}(i) .
$$

Here, $j, j^{\prime}, \ldots, j^{\prime \prime} \in p(i)$. If the amount of voltage pass defined in Condition 3 is substituted into (9) and we use $\gamma_{i j}=\gamma$ for all $j \in p(i)$ and $F_{i}\left(u_{i}\right)=y_{i} \approx u_{i}$, we get

$$
\begin{array}{r}
u_{i}-\gamma\left(u_{i}-u_{j}\right)-\gamma\left(u_{i}-u_{j^{\prime}}\right)-\cdots-\gamma\left(u_{i}-u_{j^{\prime \prime}}\right) \\
\geq u_{j^{* *}}+\gamma\left(u_{i}-u_{j^{* *}}\right) .
\end{array}
$$

We see in (10) that parameter $\gamma$ functions as a scaling factor. It affects all voltage passes from the $i$ th bulb to its neighbors; therefore, the choice of this parameter will define if (8) will hold or not. To understand how to select the parameter $\gamma$, consider the extreme case where $y_{j}=y_{j^{\prime}}=\cdots=y_{j^{\prime \prime}}$ (i.e., all neighbors of zone $i$ has the same light level). In this case, the inequality in (10) becomes

$$
u_{i}-N_{i} \gamma d \geq u_{j^{* *}}+\gamma d
$$

where $N_{i}=|p(i)|$ and $d=u_{i}-u_{j}$ for all $j \in p(i)$. With manipulation, it yields

$$
d=u_{i}-u_{j^{* *}} \geq\left(N_{i}+1\right) \gamma d .
$$

To satisfy this inequality, $\gamma$ should be selected as $\gamma \leq 1 /\left(N_{i}+\right.$ 1). In the testbed, considering that a zone connected to the network has at most three neighbors according to Fig. 15, if $\gamma \leq 1 / 4$, then Condition 2 will hold for full height partitions case. If event $e_{k} \in g\left(y_{k}\right)$ and $e_{\alpha(i)}^{i, p(i)} \in e_{k}$, then $f_{e_{k}}\left(y_{k}\right)=y_{k+1}$, where

$$
F_{i}^{\prime}=F_{i}\left(u_{i}-\sum_{\{j: j \in p(i)\}} \alpha_{j}(i)+\sum_{\left\{j: i \in p(j), e_{\alpha}(j)^{j, p(j)}\right\} \in e_{k}} \alpha_{i}(j)\right) .
$$

It might be also useful to see this relationship from bulb voltages point of view

$$
u_{i}^{\prime}=u_{i}-\sum_{\{j: j \in p(i)\}} \alpha_{j}(i)+\sum_{\left\{j: i \in p(j), e_{\alpha}(j)^{j, p(j)}\right\} \in e_{k}} \alpha_{i}(j) .
$$

The $i$ th zone bulb voltage at time $k+1, u_{i}^{\prime}$, is the voltage at time $k$ minus the total voltage passed by bulb $i$ at time $k$.

Let $E_{v}=E$ be the set of valid event trajectories. We must further specify the sets of allowed event trajectories. Define a partial event of type $i$ to represent the passing of $\alpha(i)$ amount of voltage from $i \in Z$ to its neighbors $p(i)$. A partial event of type $i$ will be denoted by $e^{i, p(i)}$ and the occurrence of $e^{i, p(i)}$ indicates that $i \in Z$ attempts to further balance its light level with its neighbors. Event $e_{k} \in g\left(y_{k}\right)$ is composed of a set of partial events. Next we define the allowed event trajectories $E_{a}$. For $E_{i} \subset E_{v}$, assume that each type of partial event occurs infinitely often on each $E \in E_{i}$.

Clearly, $\mathcal{Y}_{b}=\left\{y_{k} \in \mathcal{Y}: y_{i}=y_{j}\right\}$, for all $(i, j) \in Z$ is an invariant set that represents perfectly balanced light levels across the testbed. Notice that the only $e_{k} \in g\left(y_{k}\right)$, when $y_{k} \in$ $\mathcal{Y}_{b}$, are ones such that all $e_{\alpha(i)}^{i, p(i)} \in e_{k}$ have $\alpha(i)=(0,0, \ldots, 0)$ (i.e., there is no more voltage transfer between the bulbs when the uniform lighting is acquired in the testbed).

2) Asymptotic Convergence to a Balanced State: To study the ability of the system to automatically redistribute the bulb voltages to achieve uniform lighting across the testbed, a Lyapunov stability theoretic approach will be used. Let $S\left(\mathcal{Y}_{b} ; r\right)$ be an $r$-neighborhood of $\mathcal{Y}_{b}$ where $r>0$. Let the value of the function $Y\left(y_{o}, E_{k}, k\right)$ be the state reached at time $k$ from $y_{0} \in \mathcal{Y}$ by application of event sequence $E_{k}$ such that $E_{k} E \in E_{v}\left(y_{0}\right)$ where $E_{v}\left(y_{0}\right)$ is the set of valid event trajectories when the initial state is $y_{0} \in \mathcal{Y}$. Let $\bar{y}=\left[\bar{y}_{1}, \bar{y}_{2}, \ldots, \bar{y}_{N}\right]^{T}$. Choose

$$
\begin{array}{r}
\rho\left(y_{k}, \mathcal{Y}_{b}\right)=\inf \left\{\operatorname { m a x } \left\{\left|y_{1}-\bar{y}_{1}\right|,\left|y_{2}-\bar{y}_{2}\right|, \ldots,\right.\right. \\
\left.\left.\left|y_{N}-\bar{y}_{N}\right|\right\}: \bar{y} \in \mathcal{Y}_{b}\right\} .
\end{array}
$$

Theorem 1: For the network described above, the invariant set $\mathcal{Y}_{b}$ is asymptotically stable in the large with respect to $E_{i}$. Finally, note that modeling and stability analysis for the case where the partitions are at half height, or there are no partitions at all, is more involved than the above analysis due to the coupling. We leave such analysis as a future direction.

\section{IBA: Regulation Problem}

Several groups of experiments are carried out in this section to test the performance of the IBA. In all groups, the system is released from different initial light voltages while keeping the sum of them constant throughout the experiments. It is important to note here that it is not known what the balanced light level will be if it can be attained. The only anticipated behavior is that if the sum of applied bulb voltages is set to a greater (less) value, then the balanced light level would be higher (smaller). There is no desired light level in these regulation problem experiments; the only aim is to obtain uniform lighting across the testbed by passing voltages between the lights. We refer to this as a regulation problem although there is no reference light level here. Moreover, this 


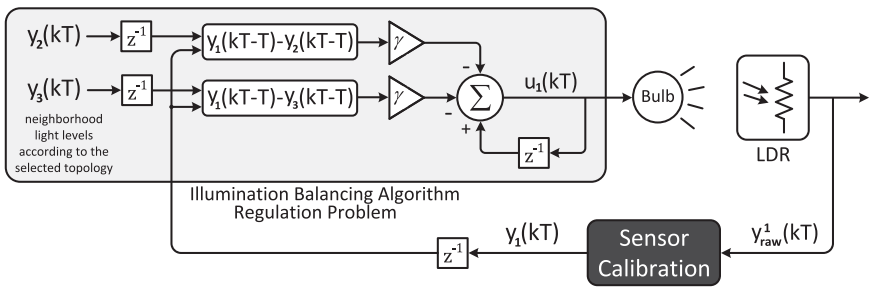

Fig. 16. Block diagram of the Zone 1 closed loop in the regulation problem. Notice that as $p(1)=\{2,3\}$ according to Fig. 15, the light levels of these zones (i.e., $y_{2}$ and $y_{3}$ ) are used as well as the Zone 1 light level $y_{1}$ in generating the bulb 1 voltage signal $u_{1}$.

reference signal should be zero for the system to be called as a regulation problem [17]. However, when the same total applied voltage is set for experiments with different initial conditions, we expect to see the balanced light level value always the same. We think of it regulating to this final balanced light level. Hence, the terminology regulation problem makes sense.

As informative examples among the experiments made for this section, two experiments with initial conditions of $u(0)=$ $[3,5,3,5,6,5,7,4]^{T}$ and $u(0)=[7,7,7,7,5,5,0,0]^{T}$ are selected. The first one has relatively distributed initial bulb voltages while the other one is obviously not distributed. However, both of the implementations satisfy $\sum_{i=1}^{8} u_{i}=38$ throughout the experiments. The value of $38 \mathrm{~V}$ is selected as it results in balanced illuminations in the linear region for all three partition cases. For the first five seconds of all experiments, initial bulb voltages were held constant to show that the light levels of the zones are initially unbalanced. Then at $t=5 \mathrm{~s}$, the IBA is turned on.

Manipulating the definition of the voltage to be passed between the bulbs given in Condition 3 (see Section IV-B) with (14) yields the following expression that describes the IBA for the regulation problem:

$$
u_{i}^{\prime}=u_{i}-\sum_{j \in p(i)} \gamma\left(y_{i}(k T)-y_{j}(k T)\right) .
$$

The control loop of Zone 1 according to (16) is depicted in Fig. 16.

The decentralized integral control already achieved desired uniform lighting across the testbed for full and half height partitions; therefore, our main concern will be no partitions case naturally. In addition to this, due to lack of space, the experiments for the first two cases will not be shown here. Nonetheless, a brief summary of these skipped experiments is given for a complete picture of the regulation problem case.

The IBA achieved uniform lighting for both of the full and half height partitions in each experiment. The balanced light levels are acquired as approximately 4.75 and $5.1 \mathrm{~V}$, respectively. This increment makes sense due to cross-illumination effects in the half height partitions case. Meanwhile, the light voltages converged to the same values despite their release from very different initial conditions.

1) Regulation Problem in No Partitions Case: The results that will be shown in this section are very important since this was the case where the decentralized integral control failed in Section III. The cross-illumination effects proved how crucial they are in no partitions case in Figs. 11 and 12. It becomes

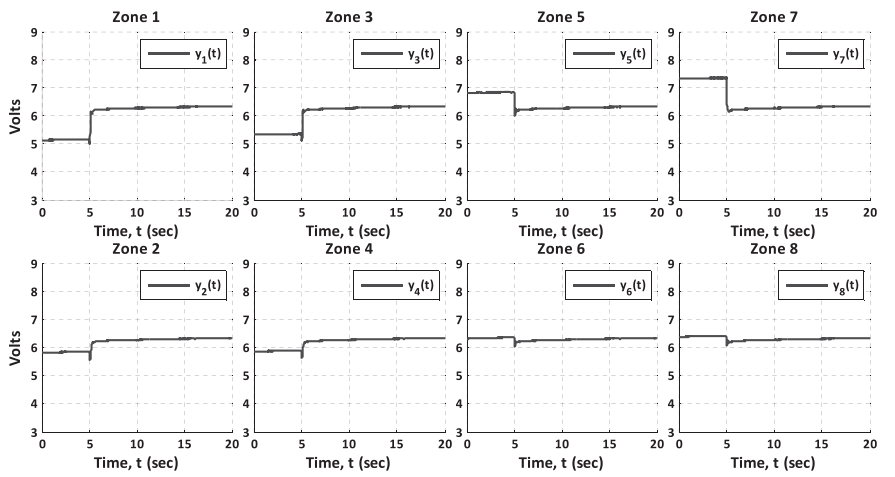

Fig. 17. Actual light levels (regulation problem, no partitions, $\gamma=0.02$ ).

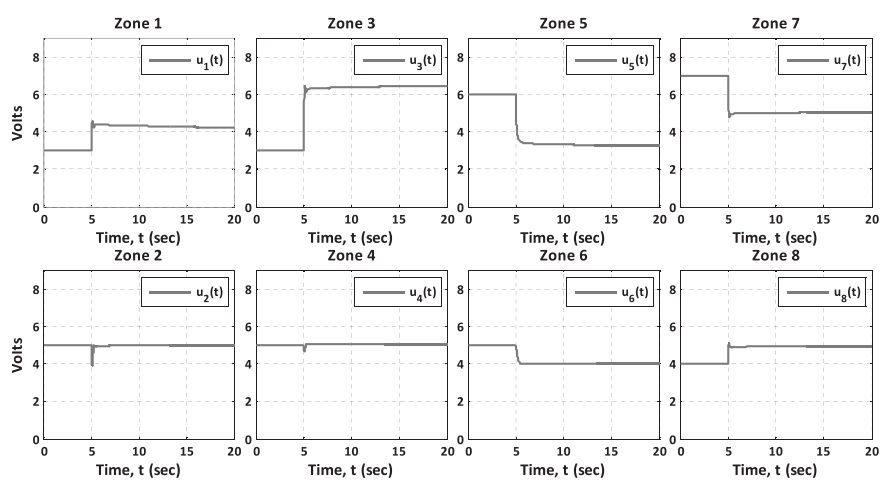

Fig. 18. Voltage signals applied to bulbs (regulation problem, no partitions, $\gamma=0.02)$.

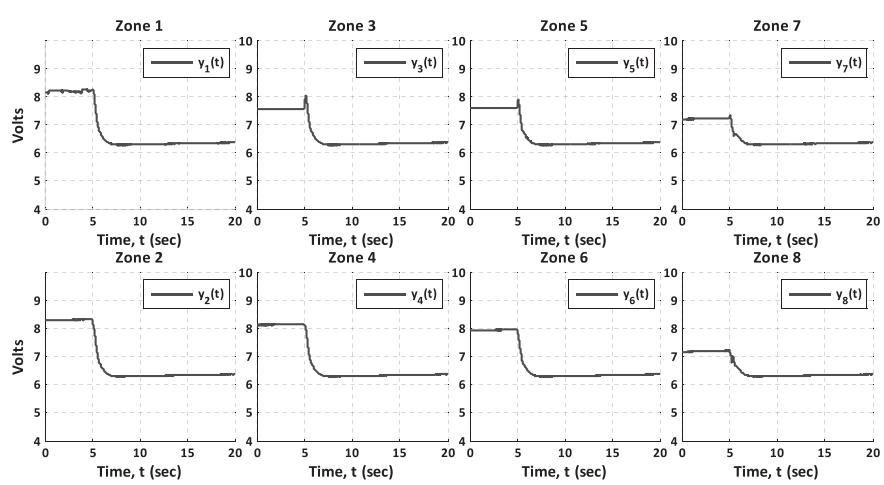

Fig. 19. Actual light levels (regulation problem, no partitions, $\gamma=0.02$ ).

impossible to predict the system response when the crossillumination effects are maximized. The results are given in Figs. 17-20, respectively, where $\gamma=0.02$.

In Figs. 17 and 19, the illuminations are balanced at about 6.35 consistently. The applied bulb voltages that result in uniform lighting are

$$
u^{*} \approx[4.25,4.98,6.42,5.09,3.27,3.95,5.05,4.97]^{T}
$$

in Figs. 18 and 20. This is a remarkable result showing that whatever the initial conditions of the system are, the IBA always balances the illumination by converging to the same applied voltage values $u^{*}$. In the previous two partition cases, the solutions were consistent as well. However, the final applied bulb voltages are not close to each other here unlike the previous cases. Meanwhile, the increments from 4.75 to 5.1, and from 5.1 to 6.35 have confirmed the increment in the 

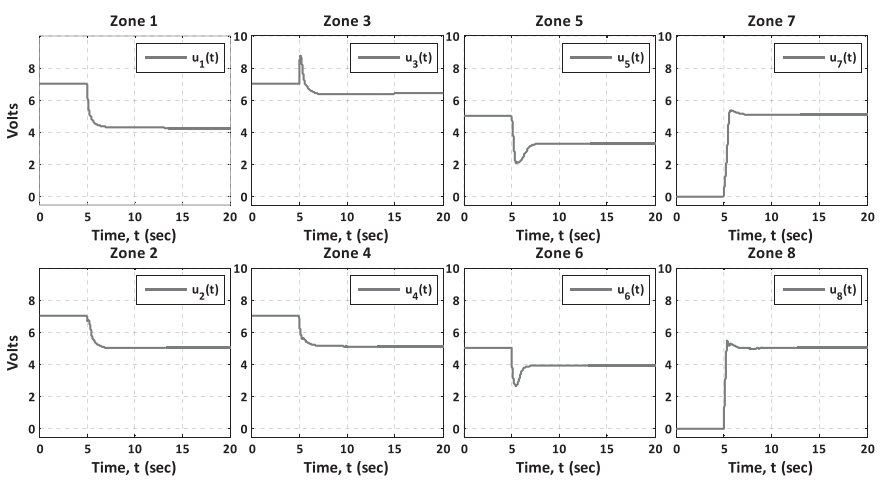

Fig. 20. Voltage signals applied to bulbs (regulation problem, no partitions, $\gamma=0.02)$.

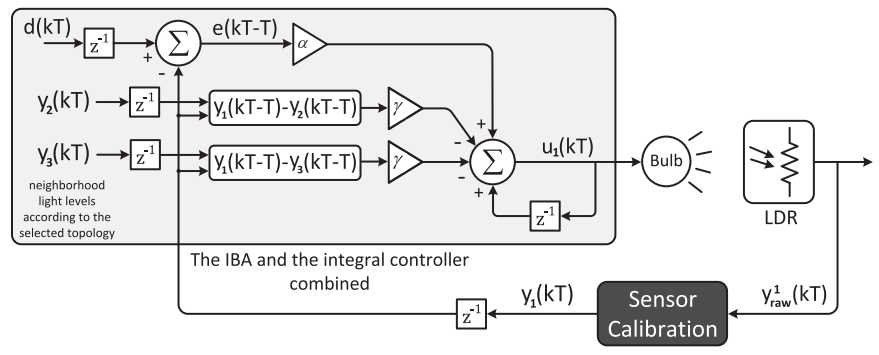

Fig. 21. Block diagram of the Zone 1 closed loop in the tracking problem.

cross-illumination effects from another perspective as the cases are switched from full height to half height, and from half height to no partitions, respectively. Consequently, in spite of the domination of the cross-illumination effects on the system behavior, the IBA achieved uniform lighting across the testbed by converging to the same light voltages every time.

\section{IBA: Tracking Problem}

In this section, the goal is not only balancing the light levels (i.e., regulation) but also tracking a desired light level. The IBA derived for the regulation problem (16) will remain running here. Furthermore, it should be improved in a way that it can decide to raise, reduce, or preserve the overall light voltage when required. The most convenient way to achieve this is to combine the heuristic algorithm developed in Section III with the IBA. Notice that it is sufficient to run the integral controller in a single zone as long as the speed of the IBA is higher than the speed of the integral controller. Otherwise, some fluctuations might occur in bulb voltages and so the light levels. The IBA will run simultaneously with the integral controller in Zone 1, which is selected arbitrarily. The expression of the algorithm for the zone 1 is given as

$$
u_{1}^{\prime}=u_{1}+\alpha e_{1}-\sum_{j \in p(1)} \gamma\left(y_{1}(k T)-y_{j}(k T)\right)
$$

while (16) applies to the rest of the zones. Here, parameter $\alpha=K_{i} T$ as given in (6) and $e_{1}$ denotes the error signal for the zone 1. The control loop for zone 1 is shown in Fig. 21.

Due to the integral controller, Zone 1 functions as an external voltage transfer gate (i.e., if the testbed is underilluminated, Zone 1 increases bulb 1 voltage, which is distributed to the rest of the zones via IBA. On the contrary,

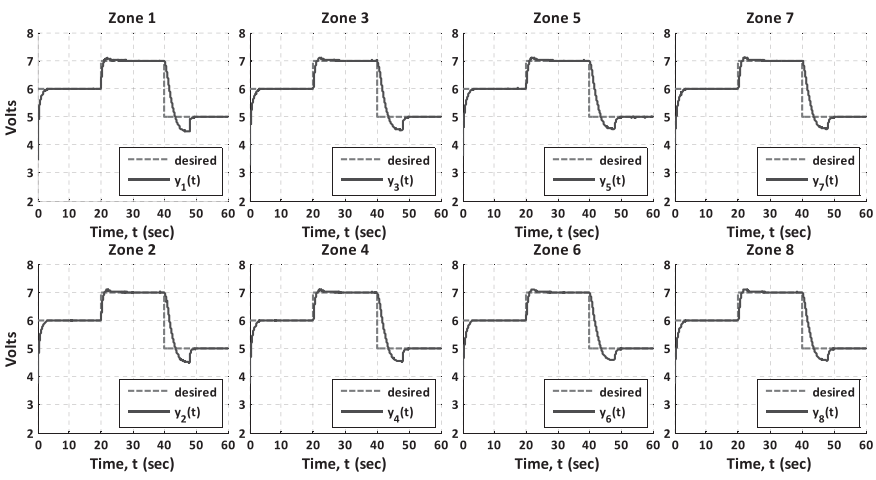

Fig. 22. Actual light levels (tracking problem, no partitions, $\gamma=0.02$, $\alpha=0.008)$

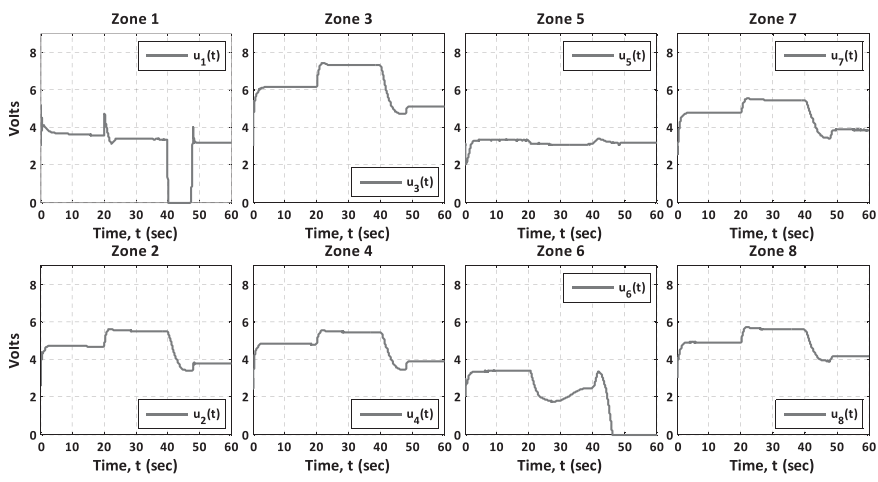

Fig. 23. Voltage signals applied to bulbs (tracking problem, no partitions, $\gamma=0.02, \alpha=0.008$ ).

if the testbed is over-illuminated, the integral controller will dim the light 1 , and the IBA will cancel this imbalance). In the following experiments, $\gamma$ will be set to 0.02 as this selection resulted in very quick convergence in the regulation problem. The value of $\alpha$ is tuned to 0.008 . This choice makes sense when $\alpha=0.001$ is recalled for the individual zones in Section III. The initial bulb voltages are set to $u(0)=[3,3,3,3,3,3,3,3]^{T}$.

Similar to the regulation problem section, we skip showing the results for full and half height partitions, including daylighting experiments (half height partitions) due to lack of space and the success of the decentralized integral control in reaching the goals before. As a summary, the combined algorithm achieved desired uniform lighting in full and half height partitions very similar to decentralized integral control results. Likewise, desired uniform lighting is preserved under variations in the external light successfully. Repeating the success of the decentralized integral control in full and half height partitions cases (Sections III-B1, III-B2, and III-B3) along with the achievement of the IBA in the regulation problem for no partitions (see Section IV-C1) is promising for the combined algorithm in no partitions case.

1) Tracking Problem in No Partitions Case: The algorithm in (18) is developed in order to overcome the failure in Section III for this case. We hope exploiting local communications between the lights will defeat the negative effects of the maximized cross-illuminations. The results are given in Figs. 22 and 23. 
As can be seen in Fig. 22, the actual light levels have shown no overshoot, a little overshoot, and some undershoot while the steps in the desired light level signal occurred at $t=0, t=20$, and $t=40$, respectively. Yet the light levels tracked the desired light level in all the zones. As a matter of fact, the IBA worked so fast that it canceled the imbalance that the integral control creates rapidly and hence all the light levels moved together. The small overshoot and the undershoot in Fig. 22 indicate the effects of the maximized couplings. Similar to the solution $u^{*}$ in Section IV-C.1, the applied bulb voltages that yield the desired uniform lighting (i.e., solutions) are not close to each other in Fig. 23.

The combined algorithm in (18) has repeated the success of the decentralized integral control in full and half height partitions cases. Moreover, it achieved desired uniform lighting in the no partitions case. The IBA uses local communications between the lights and this helped the controller to achieve uniform lighting across the testbed in the no partitions case, something that was not possible using the decentralized integral control. It is interesting that the goal of uniform lighting across the entire testbed was achieved without a centralized controller that requires global communications.

\section{CONClusion}

In this paper, we presented experimental studies on a smart lighting testbed. As the motivation of the research is to acquire a desired uniform lighting across the floor with as little information as possible, initially, a decentralized integral control was implemented that does not have any communication between the lights. All goals, including daylighting were reached in the testbed with this method except achieving uniform lighting in the case where the cross-illumination effects were maximized. In order to avoid negative effects of these high cross-couplings on separate control loops, local light level information was utilized by each individual controller in the IBA. This method achieved all the goals in the testbed without requiring a global communication between the lights. As a consequence, energy consumption was reduced by taking advantage of all light contributions and external light while providing the same light level to each zone.

\section{APPENDIX \\ PRoOF OF THEOREM 1}

Proof [16]: Choose a Lyapunov function

$$
V\left(y_{k}\right)=\max _{i}\left\{\frac{1}{N} \sum_{j=1}^{N} y_{j}-y_{i}\right\}
$$

$V\left(y_{k}\right)$ always takes positive values except in a uniform lighting situation where it becomes zero. Mathematically, $V\left(y_{k}\right) \geq$ $0, \forall y_{k}$. Notice that

$$
\frac{1}{N} \sum_{i=1}^{N} y_{i} \geq \frac{1}{N}\left(\max _{i}\left\{y_{i}\right\}+(N-1) \min _{i}\left\{y_{i}\right\}\right) .
$$

It is clear from (15), (19), and (20) that the following relations are valid:

$$
\begin{aligned}
\rho\left(y_{k}, \mathcal{Y}_{b}\right) & \geq \frac{1}{2}\left(\max _{i}\left\{y_{i}\right\}-\min _{i}\left\{y_{i}\right\}\right) \\
\rho\left(y_{k}, \mathcal{Y}_{b}\right) & \leq \max _{i}\left\{y_{i}\right\}-\min _{i}\left\{y_{i}\right\} \\
V\left(y_{k}\right) & =\frac{1}{N} \sum_{i=1}^{N} y_{i}-\min _{i}\left\{y_{i}\right\} \\
& \leq \max _{i}\left\{y_{i}\right\}-\min _{i}\left\{y_{i}\right\} \\
V\left(y_{k}\right) & \geq \frac{1}{N}\left(\max _{i}\left\{y_{i}\right\}+(N-1) \min _{i}\left\{y_{i}\right\}\right)-\min _{i}\left\{y_{i}\right\}
\end{aligned}
$$

Equations (21) and (23) yield $2 \rho\left(y_{k}, \mathcal{Y}_{b}\right) \geq \max _{i}\left\{y_{i}\right\}-$ $\min _{i}\left\{y_{i}\right\} \geq V\left(y_{k}\right)$. Equation (24) can be manipulated to yield

$$
V\left(y_{k}\right) \geq \frac{1}{N}\left(\max _{i}\left\{y_{i}\right\}-\min _{i}\left\{y_{i}\right\}\right)
$$

Equations (22) and (25) directly imply that

$$
V\left(y_{k}\right) \geq \frac{1}{N} \rho\left(y_{k}, \mathcal{Y}_{b}\right) .
$$

We must also show that $\operatorname{V}\left(Y\left(y_{o}, E_{k}, k\right)\right)$ is a nonincreasing function for all $k \in \mathbb{N}$, all $y_{0} \in S\left(\mathcal{Y}_{b} ; r\right)$ and all $E_{k}$, such that $E_{k} E \in E_{i}\left(y_{0}\right)$. To see that this is the case, notice that once $y_{0}$ is specified, $V\left(y_{k}\right)$ varies only when $\min _{i}\left\{y_{i}\right\}=y_{j^{* *}}$ varies. This least illuminated zone in the network cannot possibly pass voltage, so $y_{j^{* *}}^{\prime} \geq y_{j^{* *}}$. Assume an event $e_{k} \in g\left(y_{k}\right)$ occurs. According to Condition (2) on $e_{k} \in g\left(y_{k}\right)$, if $e_{\alpha(i)}^{i, p(i)} \in e_{k}$ and $j^{* *} \in p(i)$, it is not possible that $y_{i}^{\prime}<y_{j^{* *}}+\alpha_{j^{* *}}(i)$. Therefore, $\min _{i}\left\{y_{i}^{\prime}\right\} \geq y_{j^{* *}}^{\prime}$ and $V\left(y_{k+1}\right) \leq V\left(y_{k}\right)$. Thus, $\mathcal{Y}_{b}$ is stable in the sense of Lyapunov with respect to $E_{i}$.

In order to show that $\mathcal{Y}_{b}$ is asymptotically stable in the large with respect to $E_{i}$, it must be shown that for all $y_{0} \notin \mathcal{Y}_{b}$ and all $E_{k}$ such that $E_{k} E \in E_{i}\left(y_{0}\right), V\left(Y\left(y_{0}, E_{k}, k\right)\right) \rightarrow 0$ as $k \rightarrow \infty$. If $y_{k} \notin \mathcal{Y}_{b}$, then there must be some least illuminated zone $j^{* *}$ (there may be more than one) and some other zone $i$ such that $\left(i, j^{* *}\right) \in A$ and $y_{i}>y_{j^{* *}}$. Because of the restrictions imposed by $E_{i}$, we know that all the partial events are guaranteed to occur infinitely often. According to Condition (3) on $e_{k} \in$ $g\left(y_{k}\right)$, each time partial event $e^{i, p(i)}$ occurs, $y_{j * *}$ is guaranteed to increase by a fixed fraction $\gamma_{i j * *} \in(0,1)$ of $y_{i}-y_{j^{* *}}$ so that $y_{j^{* *}}^{\prime}>y_{j^{* *}}$. Thus, for every $k \geq 0$, there exists $k^{\prime}>k$ such that $V\left(y_{k^{\prime}}\right)>V\left(y_{k^{\prime}+1}\right)$ as long as $y_{k^{\prime}} \notin \mathcal{Y}_{b}$ so that $V\left(Y\left(y_{0}, E_{k}, k\right)\right) \rightarrow 0$ as $k \rightarrow \infty$ and $\mathcal{Y}_{b}$ is asymptotically stable in the large with respect to $E_{i}$.

\section{REFERENCES}

[1] (2011, Oct. 13). ENERGY STAR - U.S. Environmental Protection Agency \& the U.S. Department of Energy [Online]. Available: http:// www.energystar.gov/index.cfm?c=business.EPA_BUM_CH6_Lighting

[2] (2011, Oct. 11). U.S. Energy Information Administration [Online]. Available: http://www.eia.gov

[3] (2012, Aug. 21). U.S. Department of Energy, Energy Efficiency and Renewable Energy, Buildings Energy Data Book [Online]. Available: http://buildingsdatabook.eren.doe.gov 
[4] C. DiLouie, (2006). Advanced Lighting Controls: Energy Savings, Productivity, Technology and Applications. Fairmont, Boca Raton, FL, USA, [Online]. Available: http://books.google.com/books?id=XbyImaV$1 \mathrm{CsC}$

[5] F. Rubinstein, M. Siminovitch, and R. Verderber, "Fifty percent energy savings with automatic lighting controls," IEEE Trans. Ind. Appl., vol. 29, no. 4, pp. 768-773, Jul.-Aug. 1993.

[6] Y.-J. Wen and A. Agogino, "Wireless networked lighting systems for optimizing energy savings and user satisfaction," in Proc. Wireless Hive Netw. Conf., Aug. 2008, pp. 1-7.

[7] Y.-J. Wen and A. Agogino, "Control of a wireless-networked lighting system in an open-plan office," J. Lighting Res. Technol., vol. 43, pp. 235-248, Jun. 2011.

[8] V. Singhvi, A. Krause, C. Guestrin, J. Garrett, and H. S. Matthews, "Intelligent light control using sensor networks," in Proc. ACM Conf. Embedded Netw. Sensor Syst., Nov. 2005, pp. 218-229.

[9] D. T. Delaney, G. M. O'Hare, and A. G. Ruzzelli, "Evaluation of energy-efficiency in lighting systems using sensor networks," in Proc. 1st ACM Workshop Embedded Sens. Syst. Energy-Efficiency Buildings, 2009, pp. 61-66.

[10] L. Doulos, A. Tsangrassoulis, and F. Topalis, "Evaluation of lighting controls in office buildings," in Proc. 6th WSEAS Int. Conf. Circuits, Syst., Electron., Control Signal Process., Dec. 2007, pp. 69-77.

[11] A. Sarkar, M. Fairchild, and C. Salvaggio, "Integrated daylight harvesting and occupancy detection using digital imaging," in Proc. SPIE, vol. 6816, pp. 1-12, 2008.

[12] F. Hollich, The Influence of Ocular Light Perception on Metabolism in Man and in Animal. New York, NY, USA: Springer-Verlag, 1979.

[13] L. Heschong, R. Wright, and S. Okura, "Daylighting impact on retail sales performance," J. Illuminating Eng. Soc., vol. 8, no. 3, pp. 45-52, 2002.

[14] A. Galasiu and J. Veitch, "Occupant preferences and satisfaction with the luminous environment and control systems in daylit offices: A literature review," Energy Buildings, vol. 38, no. 7, pp. 728-742, 2006.

[15] D. Bertsekas and J. Tsitsiklis, Parallel and Distributed Computation: Numerical Methods. Bellmont, MA, USA: Athena Scientific, 1997.

[16] K. Passino and K. Burgess, Stability Analysis of Discrete Event Systems. New York, NY, USA: Wiley, 1998.

[17] K. Ogata, Modern Control Engineering , 4th ed. Saddle River, NJ, USA: Prentice Hall, 2002.

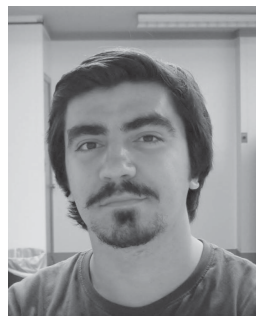

Muhammed Taha Koroglu received the M.S. degree in electrical and computer engineering from The Ohio State University (OSU), Columbus, OH, USA, in 2012. He is currently pursuing the Ph.D. degree in electrical and computer engineering as a member of the PCV Laboratories, OSU.

His current research interests include smart lights, decentralized control systems, load balancing algorithms, object detection and tracking in image streams, visual navigation, and sensor fusion.

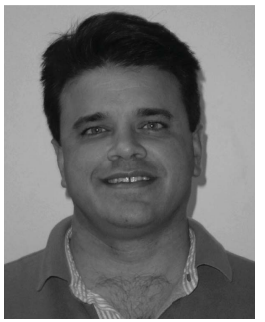

Kevin M. Passino (S'79-M'90-SM'96-F'04) received the $\mathrm{Ph} . \mathrm{D}$. degree in electrical engineering from the University of Notre Dame, South Bend, IN, USA, in 1989.

$\mathrm{He}$ is currently a Professor of electrical and computer engineering and the Director of the Center for Energy, Sustainability, and Environment, The Ohio State University, Columbus, OH, USA. He is co-editor (with P.J. Antsaklis) of the book An Introduction to Intelligent and Autonomous Control (Kluwer, 1993), co-author (with S. Yurkovich) of the book Fuzzy Control (Addison Wesley, 1998), co-author (with K.L. Burgess) of the book Stability Analysis of Discrete Event Systems (Wiley, 1998), co-author (with V. Gazi, M.L. Moore, W. Shackleford, F. Proctor, and J.S. Albus) of the book The RCS Handbook: Tools for Real Time Control Systems Software Development (Wiley, 2001), co-author (with J.T. Spooner, M. Maggiore, R. Ordonez) of the book Stable Adaptive Control and Estimation for Nonlinear Systems: Neural and Fuzzy Approximator Techniques (Wiley, 2002), author of Biomimicry for Optimization, Control, and Automation (Springer-Verlag, 2005), and co-author (with V. Gazi) of the book Swarm Stability and Optimization (Springer-Verlag, 2011).

Dr. Passino was the Director of the OSU Collaborative Center of Control Science that was funded by AFOSR and AFRL/VA. He has served as the Vice President of Technical Activities of the IEEE Control Systems Society, an elected member of the IEEE Control Systems Society Board of Governors, the Program Chair of the 2001 IEEE Conference on Decision and Control, and is currently a Distinguished Lecturer for the IEEE Control Systems Society and the IEEE Society on Social Implications of Technology. 